\title{
SUPLEMENTAC̄̃O DE VITAMINA C EM RAÇÕES PARA REVERSÃO SEXUAL DA TILÁPIA DO NILO
}

\author{
Glaucio Nogueira Toyama1,4; José Eduardo Corrente²; José Eurico Possebon Cyrino ${ }^{3 *}$ \\ ${ }^{1}$ Pós-Graduando do Depto. de Produção Animal - USP/ESALQ. \\ ${ }_{3}^{2}$ Depto. de Ciências Exatas - USP/ESALQ, C.P. 09 - CEP: 13418-900 - Piracicaba, SP. \\ ${ }^{3}$ Depto. de Produção Animal - USP/ESALQ. \\ ${ }^{4}$ Bolsista CNPq. \\ *Autor correspondente<jepcyrin@carpa.ciagri.usp.br>
}

\begin{abstract}
RESUMO: O estudo avaliou o efeito da suplementação de vitamina C: 0; 50; 100;200; 400; 600; 800; 1000 $\mathrm{mg} \mathrm{kg}^{-1}$ na dieta de reversão sexual da tilápia do Nilo (Oreochromis niloticus). Foram avaliados o ganho de peso e incremento em comprimento aos 0, 10, 20 e 30 dias de tratamento; porcentagem da sobrevivência final (S\%) e porcentagem de reversão sexual (RS\%) de pós-larvas estocadas em "hapas" de 0,08 $\mathrm{m}^{3}$. A partir do $20^{\circ}$ dia, foram observadas diferenças $(\mathrm{P}<0,0001)$ para peso e comprimento. Os melhores resultados de ganho de peso foram obtidos com suplementação acima de $800 \mathrm{mg} \mathrm{kg}^{-1}$ da dieta, e para comprimento total, níveis acima de $400 \mathrm{mg} \mathrm{kg}^{-1}$; os piores desempenhos aconteceram em níveis abaixo de $50 \mathrm{mg} \mathrm{kg}^{-1}$. Níveis de suplementação de vitamina $\mathrm{C}$ acima de $200 \mathrm{mg} \mathrm{kg}^{-1}$ proporcionaram médias semelhantes e o tratamento sem adição de vitamina $C$ proporcionou a pior $S \%$. Em relação à $R S \%$, não foram encontradas diferenças $(P<0,9993)$ entre os tratamentos. As equações de regressão polinomial para peso, comprimento total e sobrevivência, indicam que os valores de 859,5; 765,0 e 685,7 mg de vitamina C por kg da dieta, respectivamente, proporcionam os melhores resultados.
\end{abstract}

Palavras-chave: Oreochromis niloticus, vitamina C, ácido ascórbico, reversão sexual

\section{VITAMIN C DIET SUPPLEMENTATION FOR SEX REVERSAL OF THE NILE TILAPIA}

\begin{abstract}
The effects of vitamin C supplementation $\left(0 ; 50 ; 100 ; 200 ; 400 ; 600 ; 800 ; 1,000 \mathrm{mg} \mathrm{kg}^{-1}\right.$ of diet) on diets for sex reversal of the Nile tilapia, Oreochromis niloticus, were evaluated on groups of 600 larvae stocked in $0.08 \mathrm{~m}^{3}$ hapas and fed for 30 days with hormone-treated feeds containing different supplementation levels $(n=3)$. Weight gain and total length increment were evaluated after days $0,10,20$ and 30 . Survival rate (S\%) and sex reversal rate (SR\%) were determined at the end of the experimental period. Weight gain and total length became significantly different only after day $20(\mathrm{P}<0.0001)$. Best results for weight gain were attained with dietary vitamin $\mathrm{C}$ levels above $800 \mathrm{mg} \mathrm{kg}^{-1}$, while the best results for growth in length were attained with dietary vitamin C levels above $400 \mathrm{mg} \mathrm{kg}^{-1}$. Levels of supplementation higher than $200 \mathrm{mg} \mathrm{kg}^{-1}$ did not lead to better survival rates. SR\% values were not significantly affected by vitamin C supplementation of sex reversal diets $(P<0.9993)$. Optimal values for vitamin $C$ supplementation of sex reversal diets for the Nile tilapia, derived from polinomial regression analysis procedures, were: weight gain $859.5 \mathrm{mg}$; growth in length $765.0 \mathrm{mg}$, and for survival rate $685.7 \mathrm{mg}$.

Key words: Orechromis niloticus, vitamin C, ascorbic acid, sex reversal
\end{abstract}

\section{INTRODUÇÃO}

O suprimento de ovos e alevinos de qualidade e em quantidades adequadas é de fundamental importância na consolidação de uma cadeia agro-industrial na aquicultura. A obtenção de um produto de boa qualidade é diretamente relacionada com o nível de desenvolvimento tecnológico da área. Os fatores relacionados às características das espécies, ao sistema de produção e à alimentação e nutrição são os principais determinantes no sucesso da atividade.

De maneira geral, os custos associados às práticas de manejo alimentar e nutrição em piscicultura representam mais de $50 \%$ do custo total de produção em sistemas semi-intensivos, chegando a $80 \%$ em sistemas superintensivos. Alimentos mal formulados são mal aproveitados pelos peixes e podem causar sérios impactos ambientais, reduzindo o potencial de produção. Deste modo, pesquisas sobre a nutrição e determinação de níveis corretos de exigências nutricionais dos peixes constituem-se em uma importante ferramenta de desenvolvimento da piscicultura comercial, objetivando uma produção econômica e racional.

A tilápia do Nilo, embora sendo uma espécie introduzida, é sem dúvida uma das principais espécies da fauna aquática brasileira com potencial para alicerçar a expansão da piscicultura industrial. Esta espécie está devidamente adaptada às nossas condições climáticas e sua criação é feita a partir de um pacote tecnológico muito bem definido. Além disso, suas características zootécnicas e a alta qualidade de sua carne tornam-na apta ao processamento industrial e muito bem aceita pelo mercado consumidor. 
A criação de tilápias para o mercado consumidor, apresenta-se mais vantajosa quando praticada a partir de populações monosexo masculinas. Fêmeas de tilápia apresentam taxas de ganho de peso e conversão alimentar inferiores àquelas dos machos. Este fato, aliado à precocidade sexual, faz com que as fêmeas apresentem respostas menos eficientes em relação à sua produtividade quando comparadas aos machos (Lim, 1988; Lahav \& Lahav, 1990; Popma \& Lovshin, 1994). O desvio de energia para a formação dos óvulos e o período de cuidado parental com a incubação de ovos e proteção das larvas na cavidade bucal, dificulta a alimentação das fêmeas (Hepher \& Pruginin, 1981; Little et al., 1993). Além disso, a reprodução descontrolada nos sistemas de produção promove aumento exagerado da população no sistema de criação e, consequentemente, aumenta a competição por alimentos e prejudica a qualidade da água no ambiente, o que reduz ou prejudica o crescimento dos peixes.

Porque é fácil de aplicar e apresenta grande eficiência na obtenção de populações monosexo masculinas, a técnica da reversão sexual tem sido o método mais utilizado, alcançando níveis de 95 a 99\% de eficiência (Hepher \& Pruginin, 1981; Lim, 1988; Popma \& Green, 1990; Popma \& Lovshin, 1994; Pandian \& Sheela, 1995; Green et al., 1997). A reversão sexual para tilápia do Nilo consiste na administração oral de hormônio sexual masculino durante os primeiros 30 dias de vida dos peixes, fase em que ainda não exibem diferenciações sexuais secundárias. A duração do tratamento hormonal é determinada pelo consumo que, por sua vez, varia em função da temperatura da água e do estágio de desenvolvimento dos peixes (Lim, 1988; Popma \& Lovshin, 1994; Pandian \& Sheela, 1995).

O ácido ascórbico (vitamina $\mathrm{C}$ ) é uma das vitaminas essenciais para os peixes. Os peixes, assim como alguns outros animais, não conseguem sintetizar vitamina $C$ porque não apresentam a enzima Lgulonolactona oxidase que possibilita a síntese a partir da glucose (National Research Council, 1993; Soliman et al., 1986a; Steffens, 1989; Lovell, 1989).

As exigências nutricionais dos peixes por vitamina C são influenciadas por vários fatores, como idade, tamanho, estado reprodutivo, estresse, entre outros (Halver, 1985; National Research Council, 1993). Para Lim (1988) as tilápias provavelmente têm exigências metabólicas similares a outras espécies tropicais, e apresentam os sinais clássicos de deficiência de vitamina C quando esta não é suplementada na ração, ou na falta de alimento natural. O nível de inclusão de ácido ascórbico em uma dieta deve considerar a variação do material bruto, as interações entre nutrientes e não nutrientes e as perdas no processamento e estocagem.

Tilápias alimentadas com dietas sem ácido ascórbico exibem baixa taxa de crescimento, baixos índices de utilização de proteína líquida e de proteína digestível aparente, reduzida digestibilidade aparente da matéria seca, elevados níveis de umidade na composição da carcaça e baixos níveis de cinzas e proteína bruta na carcaça (Soliman et al., 1994). Podem apresentar ainda movimentos erráticos, desequilibrados e convulsivos, anorexia, redução do consumo, hemorragias ao redor da boca, olhos e nadadeiras, exoftalmia, e alta mortalidade (Soliman et al., 1986b; Shiau \& Hsu, 1995).

Soliman et al. (1994) determinaram a exigência de $125 \mathrm{mg}$ de vitamina $\mathrm{C} / 100 \mathrm{~g}$ da dieta para ótimo desenvolvimento de tilápias. Já para Popma \& Lovshin (1994) a exigência determinada para os peixes do gênero Oreochromis é de $50 \mathrm{mg} \mathrm{kg}^{-1}$ da dieta.

Este trabalho visou observar os efeitos da suplementação de vitamina $C$ no tratamento de reversão sexual da tilápia do Nilo. Esta suplementação nutricional é de grande importância nas fases iniciais de vida e do ciclo de produção da espécie, sendo fator preponderante na sustentabilidade das criações em regime intensivo, onde o uso de populações com baixa taxa de reversão sexual ou mal nutridas pode dificultar o manejo e comprometer a produtividade e a lucratividade dos sistemas.

\section{MATERIAL E MÉTODOS}

O experimento avaliou os efeitos da suplementação dietética de vitamina $C$ na fase de reversão sexual de tilápia do Nilo (Oreochromis niloticus). O experimento foi conduzido de janeiro a abril de 1998, no Setor de Piscicultura do Departamento de Produção Animal da Escola Superior de Agricultura "Luiz de Queiroz", Campus Luiz de Queiroz da Universidade de São Paulo em Piracicaba, SP.

Cada unidade experimental era constituída por um lote de 600 pós-larvas de tilápia do Nilo, provenientes da desova de reprodutores do Setor de Piscicultura da ESALQ, alojadas em hapas instalados dentro de caixas circulares de fibra de vidro de $1.000 \mathrm{~L}$, abastecidas individualmente. Os hapas foram confeccionados em malhas de poliéster revestidas com cloreto de polivinila (PVC), com abertura de malha de $1,0 \mathrm{~mm}$, com dimensões de 0,40 × 0,40 × 0,50 e volume de $0,08 \mathrm{~m}^{3}$.

A contagem das pós-larvas foi feita por comparação visual de densidade. Três lotes de 600 póslarvas foram contados e colocados em bacias plásticas iguais. Os lotes subsequentes foram estocados em bacias do mesmo tamanho, cor e quantidade de água. O número de pós-larvas de cada lote foi estimado pela observação comparativa da densidade de pós-larvas na bacia por uma única pessoa.

As larvas foram selecionadas por tamanho, conforme metodologia recomendada por Popma \& Green (1990). As ninhadas dos reprodutores foram coletadas e colocadas em uma bandeja selecionadora de malha rígida de 3,2 mm. Apenas as larvas com comprimento inferior a $13 \mathrm{~mm}$ foram utilizadas no processo de reversão sexual. O tamanho médio das larvas, no início da fase de reversão sexual foi de 0,469 $\pm 0,0707 \mathrm{mg}$ de peso e de 7,092 $\pm 0,58$ $\mathrm{mm}$ de comprimento total. 
Os tratamentos foram definidos como os níveis de suplementação de vitamina $C$ (ácido ascórbico cristalino com $95 \%$ de atividade) na ração de reversão. Foram empregados oito níveis de suplementação: $0,50,100,200$, $400,600,800$ e $1.000 \mathrm{mg}$ de vitamina $C$ por $\mathrm{kg}$ da dieta (T0; T50; T100; T200; T400; T600; T800; T1000).

A ração utilizada na fase de reversão sexual apresentava uma composição de aproximadamente $40 \%$ de proteína bruta e $3.600 \mathrm{kcal}$ de energia digestível $/ \mathrm{kg}$ de ração, tendo também suplementação mineral e vitamínica. Níveis de proteína de 25 a 50\% têm sido recomendadas na fase de larvicultura (Lim, 1988; Popma \& Green, 1990). A composição da ração utilizada foi: farinha de peixe $50 \%$; farelo de soja $25 \%$; milho integral moído $21 \%$, óleo de soja $3,8 \%$, pré-mistura mineral $0,05 \%$, pré-mistura vitamínica $0,15 \%$ e vitamina $C$, suplementada nas quantidades de $0,50,100,200,400$, 600,800 e $1.000 \mathrm{mg}$ de vitamina $\mathrm{C}$ por $\mathrm{kg}$ da dieta, definindo os tratamentos do experimento.

A suplementação vitamínica das dietas foi feita nas seguintes quantidades: vitamina $A$ 15.000.000 UI; vitamina $\mathrm{D}_{3} 1.500 .000 \mathrm{UI}$; vitamina $\mathrm{E} 60.000 \mathrm{UI}$; vitamina $\mathrm{K}_{3} 3.000 \mathrm{mg}$; vitamina $\mathrm{B}_{1} 3.000 \mathrm{mg}$; vitamina $\mathrm{B}_{2} 8.000 \mathrm{mg}$; vitamina $B_{6} 4.000 \mathrm{mg}$; vitamina $B_{12} 40 \mathrm{mg}$; ácido nicotínico $40.000 \mathrm{mg}$; ácido pantotênico $20.000 \mathrm{mg}$; ácido fólico $1.500 \mathrm{mg}$; biotina $150 \mathrm{mg}$. As dietas foram também suplementadas com minerais nas seguintes quantidades: I 1.500 mg; Co 1.000 mg; Cu 10.000 mg; Zn 100.000 mg; Fe 100.000 mg; Mn 40.000 mg.

Foi adicionado à ração de reversão, o hormônio masculino 17- $\alpha$-metiltestosterona, na dose de $60 \mathrm{mg}$ de hormônio/kg de ração. O preparo da ração de reversão seguiu a metodologia descrita por Popma \& Green (1990). As rações foram então acondicionadas em sacos plásticos de polietileno pretos e armazenadas em supercongelador. Durante o período de 30 dias de reversão, as larvas foram alimentadas 5 vezes/dia e à vontade. Dentro de cada hapa foi colocado um anel de contenção de ração flutuante, a fim de minimizar as perdas por dispersão das partículas alimentares. Os horários de alimentação foram fixados em 7:00, 9:30, 12:00, 14:30 e 17:00h.

Durante toda fase de experimentação foram monitorados os seguintes parâmetros físico-químicos da água: $\mathrm{pH}$, amônia total, oxigênio dissolvido (OD) e temperatura. Os dados de temperatura e OD foram tomados diariamente pela manhã e à tarde pelo uso de um oxímetro portátil dotado de termômetro. A variação do $\mathrm{pH}$ e dos níveis de amônia total foi monitorada semanalmente no período da tarde através de um potenciômetro e um "kit" de análise de nitrogênio amoniacal, respectivamente.

Durante o período de reversão sexual, foram amostrados lotes de 20 peixes de cada repetição, nos dias $0,10,20$ e 30 do período, e foram determinados os parâmetros biométricos de peso e comprimento total das amostras. No final da fase de reversão sexual foram avaliadas as porcentagens de sobrevivência final de cada repetição. Finalmente, foi retirada uma amostra de 50 peixes de cada repetição, que foram alojadas separadamente por tratamento em oito caixas de cimento amianto de $1.000 \mathrm{~L}$, e recriados por dois meses para verificação da porcentagem de reversão sexual por meio de seleção manual na fase juvenil. Durante esta fase de crescimento, os peixes foram alimentados com ração comercial granulada com $32 \%$ PB e $2.800 \mathrm{kcal} \mathrm{kg}^{-1}$.

$\mathrm{O}$ delineamento experimental e a conseqüente análise estatística foram realizados em esquema fatorial inteiramente casualizado, para verificar a interação de peso e comprimento total entre os oito tratamentos $(0,50,100$, $200,400,600,800$ e $\left.1.000 \mathrm{mg} \mathrm{kg}^{-1}\right)$ e as quatro datas ( 0 , 10, 20 e 30 dias). Observada a significância da interação, foi feita uma decomposição dos tratamentos dentro de cada uma das datas. Os dados de peso e comprimento total final (data 30), porcentagem de sobrevivência e porcentagem de reversão sexual foram submetidos a análises de variância e ao teste de comparação de médias de Tukey. Foram então calculadas curvas de regressão não linear para sobrevivência, porcentagem de reversão sexual, peso e comprimento total final.

\section{RESULTADOS E DISCUSSÃO}

Os parâmetros de qualidade de água monitorados durante o experimento - temperatura, OD, amônia total e $\mathrm{pH}$, mantiveram-se em níveis adequados para o conforto da espécie (Popma \& Lovshin, 1994). A temperatura média diária permaneceu acima de $25^{\circ} \mathrm{C}$; os níveis de $\mathrm{OD}$ na água foram superiores a $4,25 \mathrm{mg} \mathrm{L}^{-1}$; os valores da amônia total e do $\mathrm{pH}$ se mantiveram dentro dos parâmetros recomendados para criação de peixes: $0,01 \mathrm{mg} \mathrm{L}^{-1}$ para amônia e para pH valores entre 5 e 6 (Colt, 1991).

Os dados referentes aos incrementos em peso (P) e comprimento total (CT) médios estimados nas datas 0, 10, 20 e 30 dias do experimento estão apresentados na TABELA 1. Os resultados da análise de variância mostraram que houve interação significativa $(P<0,0001)$ entre os oito tratamentos e as quatro datas, tanto para peso como para comprimento total. A decomposição da interação tratamentos $x$ datas indicou que havia interação significativa, ao nível de $5 \%$, entre os tratamentos, nas datas 20 e 30 , para peso $(P<0,0001$ e $P<0,0001)$ e comprimento total $(P<0,0001$ e $P<0,0001)$.

A análise de variância e o teste de comparação de médias de peso e comprimento total na data 30 , revelaram diferenças tanto em relação ao peso quanto em relação ao comprimento total. Os maiores incrementos em peso foram observados nos tratamentos T800 e T1000 e os piores resultados foram encontrados nos tratamentos T50 e T0 (TABELA 2). Em relação ao incremento em comprimento, os melhores resultados foram obtidos com níveis de suplementação acima $400 \mathrm{mg}$ de vitamina $\mathrm{C} \mathrm{kg}^{-1}$ da dieta (T400 a T1000) e os piores para os tratamentos T0 e T50. 
TABELA 1 - Valores médios de peso (P) e comprimento total (CT) obtidos nas biometrias realizadas durante a fase de reversão sexual da tilápia do Nilo.

\begin{tabular}{|c|c|c|c|c|c|c|c|c|}
\hline \multirow[b]{3}{*}{ Tratamento } & \multicolumn{8}{|c|}{ Tempo (dias) } \\
\hline & \multicolumn{2}{|c|}{0} & \multicolumn{2}{|c|}{10} & \multicolumn{2}{|c|}{20} & \multicolumn{2}{|c|}{30} \\
\hline & $\mathrm{P}(\mathrm{mg})$ & $\mathrm{CT}(\mathrm{mm})$ & $\mathrm{P}(\mathrm{mg})$ & $\mathrm{CT}(\mathrm{mm})$ & $P(\mathrm{mg})$ & $\mathrm{CT}(\mathrm{mm})$ & $\mathrm{P}(\mathrm{mg})$ & $\mathrm{CT}(\mathrm{mm})$ \\
\hline T0 & 0,469 & 7,092 & 8,075 & 9,094 & 48,747 & 14,300 & 269,028 & 23,597 \\
\hline$T 50$ & 0,469 & 7,092 & 8,358 & 9,013 & 49,580 & 14,698 & 288,611 & 24,875 \\
\hline T100 & 0,469 & 7,092 & 8,315 & 9,292 & 49,377 & 14,717 & 329,097 & 26,083 \\
\hline T200 & 0,469 & 7,092 & 8,113 & 9,480 & 57,002 & 15,303 & 339,861 & 26,889 \\
\hline T400 & 0,469 & 7,092 & 8,327 & 9,420 & 61,247 & 16,324 & 387,500 & 29,000 \\
\hline T600 & 0,469 & 7,092 & 8,440 & 9,497 & 64,112 & 16,460 & 401,667 & 29,153 \\
\hline T800 & 0,469 & 7,092 & 8,388 & 9,606 & 65,673 & 16,454 & 423,472 & 29,694 \\
\hline $\mathrm{T} 1000$ & 0,469 & 7,092 & 8,508 & 9,349 & 68,170 & 16,770 & 422,361 & 29,556 \\
\hline
\end{tabular}

TABELA 2 - Comparação de médias das variáveis peso e comprimento.

\begin{tabular}{lcccc}
\hline Tratamento & Peso & $\begin{array}{c}\text { Teste de } \\
\text { Tukey }\end{array}$ & Comprimento & $\begin{array}{c}\text { Teste de } \\
\text { Tukey }\end{array}$ \\
\hline T800 & 423,472 & A & 29,694 & A \\
T1000 & 422,361 & AB & 29,556 & A \\
T600 & 401,667 & B & 29,153 & A \\
T400 & 387,500 & C & 29,000 & A \\
T200 & 339,861 & D & 26,889 & B \\
T100 & 329,097 & D & 26,083 & BC \\
T50 & 288,611 & E & 24,875 & CD \\
T0 & 269,028 & F & 23,597 & D \\
\hline
\end{tabular}

Médias com a mesma letra no Grupo de Tukey não diferem a 5\%.

Os gráficos de dispersão das médias dos tratamentos e as equações de regressão polinomial para peso e comprimento total estão apresentadas nas Figuras 1 e 2. As derivações das equações mostraram que os níveis de inclusão que promoveram maior crescimento em peso e em comprimento foram 859,5 e $765,0 \mathrm{mg}$ de vitamina $\mathrm{C} \mathrm{kg}^{-1}$ da dieta, respectivamente.

As parcelas começaram a apresentar diferença de crescimento, tanto em peso quanto em comprimento total, após a terceira biometria, 20 dias após o início do experimento. Esta situação é consistente com observações de alguns pesquisadores que constataram a transferência de nutrientes, inclusive ácido ascórbico, dos reprodutores para larvas, via vitelo. Soliman et al. (1986b) com tilápia mossambica (Oreochromis mossambicus) e Blom \& Dabrowski (1996) com truta arcoíris (Oncorhynchus mykiss), estudaram o efeito da suplementação alimentar com ácido ascórbico na progênie e verificaram que a qualidade dos produtos obtidos era melhor para reprodutores alimentados com dietas suplementadas com vitamina $\mathrm{C}$, em comparação com os reprodutores alimentados com dietas livre de vitamina $C$, sugerindo a transferência de ácido ascórbico via ovo para as larvas, o que assegura alguma reserva nos estágios iniciais de vida da larva. As larvas utilizadas neste experimento foram provenientes de reprodutores saudáveis, alimentados com ração completa e confinados em viveiros de terra.

A taxa de crescimento dos peixes está relacionada a diversos fatores, englobando o ambiente de criação, a disponibilidade de alimento e as características da espécie. Deste modo, os índices de crescimento das pós-larvas de tilápias ao final do período de reversão sexual podem variar muito. De acordo com Popma \& Green (1990) e Popma \& Lovshin (1994), ao final do período de reversão sexual o peso médio dos peixes poderá ser de 100 a $500 \mathrm{mg}$, e a temperatura da água e a qualidade da ração são os fatores que mais influenciam esta variação.

Vera Cruz \& Mair (1994) avaliando a influência da densidade de estocagem no ganho de peso de tilápias ao final do período de reversão sexual, relataram ganhos de peso de 33,5, 56,2 e 145,6 mg, respectivamente, para densidades de estocagem de 10.000; 6.000 e 2.000 póslarvas $\mathrm{m}^{-3}$. Observaram ainda que o ganho de peso médio de tilápias ao final do período de reversão sexual realizada em hapas pode variar de 221,4 a 419,0 mg. A deterioração dos parâmetros de qualidade de água causada por altas densidades de estocagem na fase de reversão sexual pode interferir negativamente no crescimento dos peixes, tornando-se o principal fator de variação no ganho de peso das pós-larvas neste período (Varadaraj et al., 1994; Phelps et al., 1995).

Outros autores têm conseguido índices de crescimento superiores à faixa encontrada por Popma \& Lovshin (1994). É o caso de Varadaraj \& Pandian (1991), que obtiveram índices médios de crescimento maiores que $540 \mathrm{mg}$, com um máximo de $1.083 \mathrm{mg}$, e Phelps et al. (1995), que conseguiram um ganho de peso variando de 760 a $2.800 \mathrm{mg}$, trabalhando com diferentes densidades de estocagem em viveiros de terra.

Diversos pesquisadores têm relatado os efeitos da falta ou da utilização de níveis inadequados de ácido ascórbico na dieta em diversas espécies. Soliman et al. 
(1986b) observaram que larvas de tilápia mossambica alimentadas com dietas desprovidas de ácido ascórbico apresentam baixa taxa de crescimento específico, piores índices de conversão alimentar e taxa de eficiência protéica, e menor taxa de sobrevivência. Esses mesmos autores atribuíram a severa redução no crescimento e o pior índice de conversão alimentar obtidos das larvas alimentadas com dietas sem ácido ascórbico à anorexia e redução da atividade voluntária dos peixes (letargia).

Mahajan \& Agrawal (1980) trabalhando com larvas de carpa indiana submetidas a diferentes níveis de inclusão de vitamina $C$ na dieta, observaram que as taxas de crescimento das larvas aumentaram proporcionalmente ao aumento da quantidade de ácido ascórbico na dieta, até um máximo de $600 \mathrm{mg} \mathrm{kg}^{-1}$. Os piores índices de crescimento das larvas foram observados nos grupos que receberam uma dieta sem suplementação de vitamina C, ou cuja dieta foi suplementada com $60 \mathrm{mg}$ de vitamina $C$ por $\mathrm{kg}$ da dieta.

Neste trabalho, os melhores índices de ganho de peso foram observados em níveis de suplementação acima de $800 \mathrm{mg}$ de vitamina $\mathrm{C} \mathrm{kg}^{-1}$ da dieta, e os melhores índices de incremento em comprimento foram observados para níveis de suplementação de vitamina $C$ acima de $400 \mathrm{mg} \mathrm{kg}^{-1}$ da dieta. A equação de regressão polinomial indica valores para ótimo desempenho de 859,5 e 765,0 mg de vitamina $\mathrm{C} \mathrm{kg}^{-1}$ da dieta, respectivamente para ganho de peso e incremento em comprimento (Figuras 1 e 2). Os alevinos obtidos ao final do experimento estavam na faixa de peso e comprimento adequados para comercialização e dentro dos padrões normais de crescimento. O peso e o comprimento total variaram de 269,03 a 423,47 mg e de 23,60 a $29,70 \mathrm{~mm}$, respectivamente. Estes resultados comprovam que 0 aumento dos níveis de suplementação com vitamina $C$ na dieta proporciona melhores índices de desempenho.

A análise de variância dos dados de $\mathrm{S} \%$ em função da variação do nível de suplementação de vitamina $C$ nas dietas indicou que houve diferença $(P<0,001)$ entre os tratamentos (TABELA 3). A aplicação

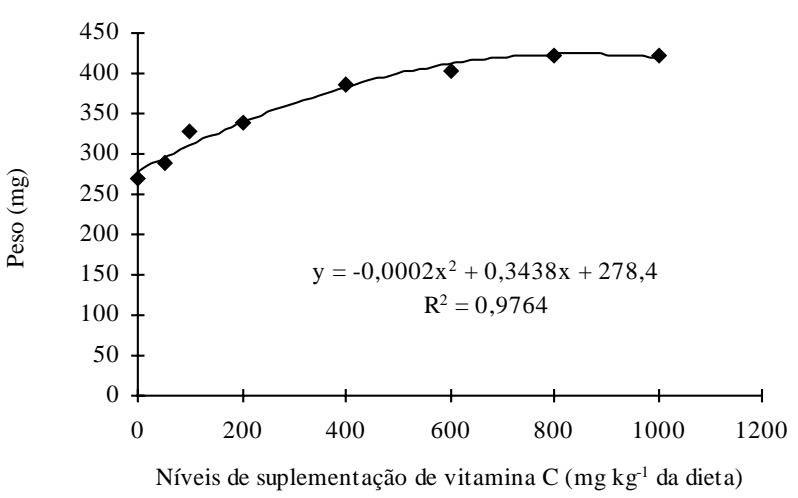

Figura 1 - Dispersão e equação de regressão polinomial para peso final, na fase de reversão sexual da tilápia do Nilo (30 dias).

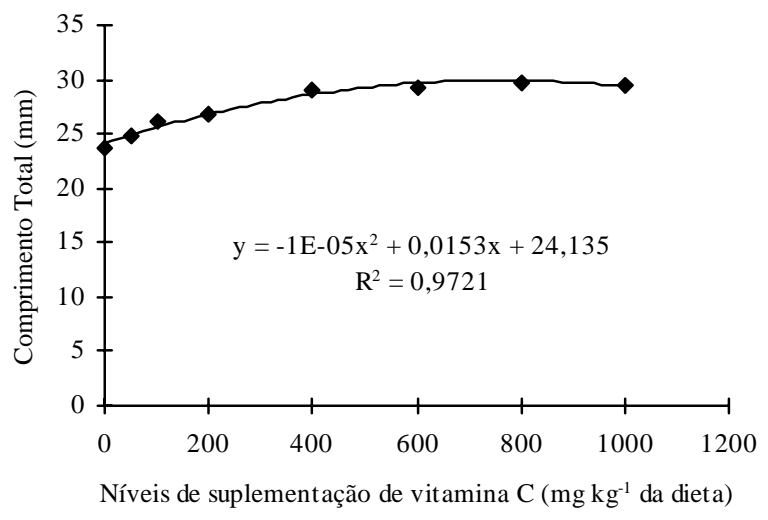

Figura 2 - Dispersão e equação de regressão polinomial para comprimento total final, na fase de reversão sexual da tilápia do Nilo (30 dias).

do teste de comparação de médias comprova a diferença significativa entre os tratamentos.

A análise de variância e 0 teste de comparação de médias dos dados de RS\% revelaram não existir diferenças $(P>0,9993)$ entre os tratamentos em relação aos valores de porcentagem de machos nas populações resultante do procedimento de reversão. As porcentagens de machos nas populações dos tratamentos variou de 89 a $91 \%$ (TABELA 3).

$O$ aumento da concentração de vitamina $C$ na dieta proporcionou maior sobrevivência média no final do experimento. Os tratamentos de T200 a T1000 apresentaram as maiores porcentagens de sobrevivência final, com valores acima de $61,6 \%$, e não apresentaram diferenças entre si. Já os tratamentos T50 e T100 apresentaram sobrevivência média final de $37,9 \%$ e $44,1 \%$, respectivamente, e o pior resultado foi observado para o tratamento T0, com $26,55 \%$ de sobrevivência média final.

O gráfico de dispersão das médias dos tratamentos e a equação de regressão polinomial para sobrevivência estão apresentados na Figura 3. A derivação da sua equação mostrou que o nível de inclusão que promoveu maior sobrevivência foi $685,7 \mathrm{mg}$ de vitamina $\mathrm{C} \mathrm{kg}^{-1}$ da dieta.

TABELA 3 - Porcentagem média de sobrevivência (S\%) e porcentagem média de reversão sexual (RS\%) por tratamento ao final da fase de reversão sexual de tilápia do Nilo.

\begin{tabular}{lcc}
\hline Tratamentos & $\mathrm{S} \%$ & $\mathrm{RS} \%$ \\
\hline T0 & 26,556 & 91 \\
T50 & 37,889 & 89 \\
T100 & 44,111 & 90 \\
T200 & 61,722 & 89 \\
T400 & 63,111 & 91 \\
T600 & 61,778 & 89 \\
T800 & 61,611 & 91 \\
\hline T1000 & 67,889 & 90 \\
\hline
\end{tabular}




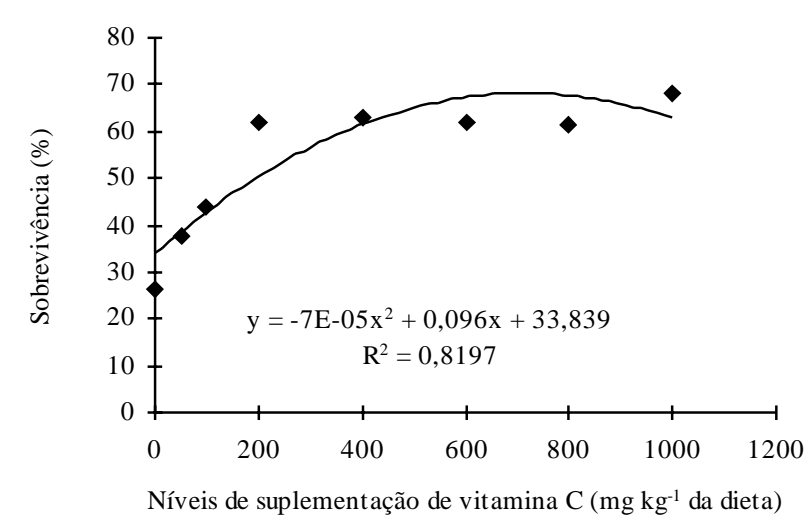

Figura 3 - Dispersão e equação de regressão polinomial para sobrevivência final, na fase de reversão sexual da tilápia do Nilo (30 dias).

A sobrevivência final no processo de reversão sexual com tilápias pode variar em função de diversos fatores. Popma \& Lovshin (1994) citaram que a sobrevivência final encontrada nestes tratamentos de reversão sexual pode ser menor que 50\%, mas normalmente está entre 70 e $80 \%$. Tais valores foram confirmados por Varadaraj \& Pandian (1991), que relataram 74 a $98 \%$ de sobrevivência; Vera Cruz \& Mair (1994) encontraram valores de 70,8 a 84,7\% em hapas; e Abucay \& Mair (1997) obtiveram sobrevivência final de 68,0 a 99,3\% com tilápias do Nilo submetidas ao tratamento de reversão sexual em hapas, a uma densidade de estocagem de 1.800 pós-larvas $\mathrm{m}^{-3}$.

A densidade de estocagem é um importante fator relacionado com a sobrevivência. Vera Cruz \& Mair (1994) constataram que o aumento da densidade de estocagem reduz a sobrevivência final de pós-larvas de tilápias submetidas à reversão sexual em hapas, e relataram valores de 92,2; 76,1 e 68,9\% de sobrevivência para densidades de estocagem de 2.000; 6.000 e 10.000 póslarvas $\mathrm{m}^{-3}$, respectivamente. Resultados semelhantes foram obtidos no trabalho de Varadaraj et al. (1994), que afirmaram que a sobrevivência foi drasticamente diminuída quando a densidade de estocagem foi maior que 500 póslarvas $\mathrm{m}^{-2}$. À luz dos resultados relatados acima, podemos inferir que os valores de S\% obtidos neste experimento estão dentro da normalidade, em função da densidade de estocagem utilizada.

Níveis inadequados de vitamina $C$ na dieta aumentam a mortandade de peixes nas fases larval e póslarval, em que apresentam ritmo de desenvolvimento mais acentuado. É muito difícil a observação dos sintomas de deficiência nutricional em larvas e pós-larvas alimentadas com dietas onde os níveis de vitamina $C$ estão abaixo das exigências nutricionais, principalmente em situações em que a disponibilidade do alimento natural é reduzida. 0 sintoma mais aparente é a mortandade. Para Fracalossi (1998) os níveis de nutrientes que podem promover um rápido crescimento não são necessariamente aqueles que
TABELA 4 - Comparação de médias das variáveis reversão sexual e sobrevivência.

\begin{tabular}{lcc}
\hline & Reversão Sexual & Sobrevivência \\
Tratam ento & Média & Média \\
\hline T0 & $0,9133 \mathrm{~A}$ & $67,889 \mathrm{~A}$ \\
T800 & $0,9133 \mathrm{~A}$ & $63,111 \mathrm{~A}$ \\
T400 & $0,9067 \mathrm{~A}$ & $61,778 \mathrm{~A}$ \\
T100 & $0,9000 \mathrm{~A}$ & $61,722 \mathrm{~A}$ \\
T1000 & $0,9000 \mathrm{~A}$ & $61,611 \mathrm{~A}$ \\
T600 & $0,8933 \mathrm{~A}$ & $44,111 \mathrm{~B}$ \\
T200 & $0,8933 \mathrm{~A}$ & $37,889 \mathrm{~B}$ \\
T50 & $0,8867 \mathrm{~A}$ & $26,555 \mathrm{C}$ \\
\hline
\end{tabular}

Médias com a mesma letra no Grupo de Tukey não diferem a 5\%.

induziriam resistência a doenças ou um fortalecimento do sistema imunológico. Os peixes podem estar deficientes em algum nutriente, sem exibir sinais clínicos de deficiência nutricional.

Soliman et al. (1994) observaram que a sobrevivência de alevinos de tilápia do Nilo é reduzida de 80 para $50 \%$ quando as dietas não são suplementadas com ácido ascórbico. Esses pesquisadores afirmaram que a mortandade observada nas populações de peixes com privação de vitamina $\mathrm{C}$ na dieta é resultado do efeito cumulativo da deficiência crônica deste nutriente em todos os processos metabólicos em que ele é essencial. Mahajan \& Agrawal (1980) também verificaram índices de mortandade de $42 \%$ e $38 \%$, respectivamente, em populações de larvas de carpa indiana (Cirrhina mrigala) alimentadas com dietas sem suplementação de ácido ascórbico ou com suplementação de $60 \mathrm{mg}$ de vitamina $\mathrm{C}$ por kg de alimento. Shiau \& Jan (1992) também verificaram que a deficiência de ácido ascórbico na dieta de juvenis de tilápias resulta na mortandade de $37,8 \%$ da população.

Neste experimento, a sobrevivência foi significativamente maior para os tratamentos com suplementação de vitamina $\mathrm{C}$ acima de $200 \mathrm{mg} \mathrm{kg}^{-1}$ - sobrevivências médias estimadas de $61,6 \%$ a $67,9 \%$, enquanto que os níveis de suplementação de 50 e $100 \mathrm{mg} \mathrm{kg}^{-1}$ apresentaram índices de sobrevivência de 37,9\% e 44,1\%, respectivamente. $O$ tratamento com o menor nível de suplementação teve o pior desempenho em relação ao índice de sobrevivência, que foi de apenas $26,5 \%$.

O índice de sucesso preconizado na reversão sexual de tilápia do Nilo - porcentagem de machos encontrada nas populações submetidas ao processo - é superior a 95\%, mas usualmente encontra-se entre $80 \mathrm{e}$ 90\% (Popma \& Lovshin, 1994). Green et al. (1997) fizeram uma revisão sobre o tema e concluíram que a administração oral de 30 a $60 \mathrm{mg}$ de metiltestosterona por kg de ração para larvas de tilápia durante um período de 3 a 4 semanas produz populações compostas por mais de $95 \%$ de machos. 
Vários autores, como Phelps et al. (1995) e Vera Cruz \& Mair (1994) relatam porcentagens de reversão sexual variando de 85 a 98,2\%, e creditam esta variação a condições de condução do processo de reversão: uso de tanques, viveiros ou hapas; e variação na densidade de estocagem. Já para Varadaraj et al. (1994) o sucesso do processo de reversão sexual e a taxa de crescimento das pós-larvas dependem diretamente da taxa diária de alimentação, que deve ser fixada em $25 \%$ do peso vivo, e não da densidade de estocagem.

As altas doses de suplementação, indicadas como resultados das equações de regressão polinomial do experimento para ótimo desempenho, são um reflexo do tipo e da composição da dieta utilizada. A ração em pó utilizada na alimentação inicial de peixes sofre altas perdas com lixiviação e a solubilização dos nutrientes na água em função da desagregação das partículas alimentares, exigindo níveis mais elevados de suplementação vitamínica.

O ácido ascórbico é um dos nutrientes cuja estabilidade é mais comprometida, sujeito a grandes perdas no processo de alimentação de peixes com rações pulverizadas (Halver, 1989; Steffens, 1989; Tacon, 1991). Para reduzir a possibilidade de ocorrência de problemas com deficiência de vitamina $C$ na dieta, é recomendada a utilização de suplementação excessiva na dieta (De Silva \& Anderson, 1995), ou a utilização de formas mais estáveis ou protegidas de ácido ascórbico, menos sujeitas a perdas por oxidação ou lixiviação (Soliman et al., 1986a; Tacon, 1991; Soliman et al., 1994; Shiau \& Hsu, 1995; 1996; Nitzan et al., 1996).

\section{CONCLUSÕES}

Não há efeito direto da suplementação da dieta com ácido ascórbico no índice de sucesso na reversão sexual da tilápia do Nilo. O ganho de peso e o incremento em comprimento total de pós-larvas de tilápia do Nilo são otimizados, respectivamente, com 765 a 859,5 mg de ácido ascórbico $\mathrm{kg}^{-1}$ da dieta, e que a suplementação de 685,7 $\mathrm{mg} \mathrm{kg}^{-1}$ permite obter melhores índices de sobrevivência de larvas submetidas a tratamento hormonal para reversão sexual.

\section{REFERÊNCIAS BIBLIOGRÁFICAS}

ABUCAY, J.S.; MAIR, G.C. Hormonal sex reversal of tilapias: implications of hormone treatment application in closed water systems. Aquaculture Research, v.28, p.841-845, 1997.

BLOM, J.H.; DABROWSKI, K. Ascorbic acid metabolism in fish: is there a maternal effect on the progeny? Aquaculture, v.147, p.215-224, 1996.

COLT, J. Aquacultural production systems. Journal of Animal Science, v.69, p.4183-4192, 1991.

DE SILVA, S.S.; ANDERSON, T.A. Fish nutrition in aquaculture. London: Chapman \& Hall, 1995. 319p.
FRACALOSSI, D.M. Doenças nutricionais em peixes. In: SIMPÓSIO SOBRE MANEJO E NUTRIÇÃO DE PEIXES, 2., Piracicaba, 1998. Anais. Campinas: CBNA, 1998. p.97-122.

GREEN, B.W.; VEVERICA, K.L.; FITZPATRICK, M.S. Fry and fingerling production. In: EGNA, H.S.; BOYD, C.E. (Ed.) Dynamics of pond aquaculture. Boca Raton: CRC Press, 1997. p.215-243.

HALVER, J.E. Recent advances in vitamin nutrition and metabolism in fish. In: COWEY, C.B; MACKIE, A.M.; BELL, J.G. (Ed.) Nutrition and feeding in fish. London: Academic Press, 1985. p.415-429.

HALVER, J.E. The vitamins. In: HALVER, J.E. (Ed.) Fish nutrition. Washington: Academic Press, 1989. p.31-109.

HEPHER, B.; PRUGININ, Y. Commercial fish farming. New York: John Willey, 1981. 261p.

LAHAV, M.; LAHAV, E. The developmentof all-male tilapia hybrids in Nir David. The Israely Journal of Aquaculture, v.42, p.58-61, 1990.

LIM, C. Pratical feeding: Tilapias. In: LOVELL, T. (Ed.) Nutrition and feeding of fish. New York: Van Nostrand Reinhold, 1988. p.163-182.

LITTLE, D.C.; MACINTOSH, D.J.; EDWARDS, P. Improving spawning synchrony in the Nile tilapia, Oreochromis niloticus (L.). Aquaculture Fisheries Management, v.24, p.399-405, 1993.

LOVELL, R.T. Nutrition and feeding of fish. New York: Van Nostrand Reinhold, 1989. 260p.

MAHAJAN, C.L.; AGRAWAL, N.K. Nutritional requirement of ascorbic acid by indian major carp, Cirrhina mrigala, during early growth. Aquaculture, v.19, p.37-48, 1980.

NITZAN, S.; ANGEONI, H.; GUR, N. Effects of ascorbic acid polyphosphate (AAPP) enrichment on growth, survival and disease resistance of hybrid tilapia. The Israeli Journal of Aquaculture, v.48, p.133-141, 1996.

NATIONAL RESEARCH COUNCIL. Nutrient requirement of fish. Washington: National Academic Press, 1993. 114p.

PANDIAN, T.J.; SHEELA, S.G. Hormonal induction of sex reversal in fish. Aquaculture, v.138, p.1-22, 1995.

PHELPS, R.P.; SALAZAR, G.C.; ABE, V.; ARGUE, B.J. Sex reversal and nursery growth of Nile tilapia, Oreochromis niloticus (L.), free-swimming in earthen ponds. Aquaculture Research, v.26, p.293-295, 1995.

POPMA, T.J.; GREEN, B.W. Aquacultural production manual: Sex reversal of tilapia in earthen ponds. Auburn: Auburn University, International Center for Aquaculture, 1990. 15p. (Research and Development Series, 35).

POPMA, T.J.; LOVSHIN, L.L. Worldwide prospects for commercial production of tilapia. Auburn: Auburn University, Center for Aquaculture and Aquatic Enviroments, Department of Fisheries and Allied Aquacultures, 1994. 40p.

SHIAU, S.-Y.; HSU, T.-S. L- Ascorbyl-2-sulfate has equal antiscorbutic activity as L-ascorbyl-2-monophosphate for tilapia, Oreochromis niloticus x O. aureus. Aquaculture, v.133, p.147-157, 1995.

SHIAU, S.-Y.; JAN, F.L. Dietary ascorbic acid requirement of juvenile tilapia Oreochromis niloticus X O. aureus. Bulletin of the Japanese Society of Scientific Fisheries, v.58, p.671-675, 1992.

SOLIMAN, A.K.; JAUNCEY, K.; ROBERTS, R.J. The effect of varying forms of dietary ascorbic acid on the nutrition of juvenile tilapias (Oreochromis niloticus). Aquaculture, v.52, p.1-10, 1986a.

SOLIMAN, A.K.; JAUNCEY, K.; ROBERTS, R.J. The effect of dietary ascorbic acid supplementation on hatchability, survival rate and fry performance in Oreochromis mossambicus (Peters). Aquaculture, v.59, p.197-208, 1986b. 
SOLIMAN, A.K.; JAUNCEY, K.; ROBERTS, R.J. Water-soluble vitamin requirements of tilapia: ascorbic acid (vitamin C) requirement of Nile tilapia, Oreochromis niloticus (L.). Aquaculture and Fisheries Management, v.25, p.269-278, 1994.

STEFFENS, W. Principles of fish nutrition. Chichester: Ellis Harwood, 1989. 384p.

TACON, A.G.J. Vitamin nutrition in shrimp and fish. In: AQUACULTURES FEED PROCESSING AND NUTRITION WORKSHOP, Singapore, 1991. Proceedings. Singapore: American Soybean Association, 1991. p.10-41.
VARADARAJ, K.; PANDIAN, T.J. Effect of solubilizing $17 \alpha-$ ethynyltestosterona in three different solvents on sex reversal of Mozambique tilapia. The Progressive Fish-Culturist, v.53, p.67-71, 1991.

VARADARAJ, K.; KUMARI, S.S.; PANDIAN, T.J. Comparison of conditions for hormonal sex reversal of Mozambique tilapias. The Progressive Fish-Culturist, v.56, p.81-90, 1994.

VERA CRUZ, E.M.; MAIR, G.C. Conditions for effective androgen sex reversal in Oreochromis niloticus (L.). Aquaculture, v.122, p.237-248, 1994.

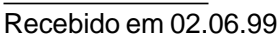

